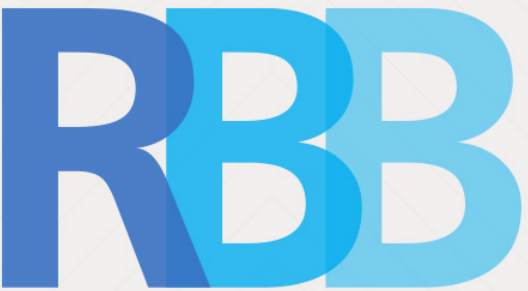

Revista Brasileira de Bioética

\author{
Márcia Farsura de Oliveira \\ Faculdade Dinâmica do Vale do \\ Piranga/FADIP, Ponte Nova, MG, \\ Brasil \\ mmfarsura@yahoo.com.br
}

Marli do Carmo Cupertino

Faculdade Dinâmica do Vale do

Piranga/FADIP, Ponte Nova, MG,

Brasil

marli.cupertino.vet@gmail.com

\section{Ensino-serviço na graduação médica: enfoque no valor da vida humana}

\author{
Teaching-service in medical school: a focus on the value of \\ human life
}

Resumo: A humanização e a ética são ferramentas essenciais para melhorar a atenção e gestão nos serviços e sistemas de saúde. Assim, este estudo objetivou analisar e evidenciar as questões éticas envolvendo as mudanças no processo de trabalho em saúde, no sistema único de saúde, a partir da Política Nacional de Humanização (PNH) de 2003 e das Diretrizes Curriculares Nacionais (DCNs) para o curso de graduação em Medicina de 2014. Como resultado observou-se que a bioética foi uma ferramenta fundamental para estabelecimento das novas DCNs e $\mathrm{PNH}$, através da construção de novas relações entre usuários e trabalhadores e destes entre si, com articulação de atitudes que garantam uma atenção integral, resolutiva e de forma humanizada. Além de auxiliar aos médicos a determinarem um elo entre o saber científico e o saber humanístico, prevenindo os malefícios que os avanços tecnológicos possam exercer sobre os seres vivos. Conclui-se que a bioética foi uma ferramenta importante para estabelecimento das novas DCNs e da $\mathrm{PNH}$.

Palavras-chave: Bioética, ensino médico, educação em saúde, humanização da assistência.

Abstract: Humanization and ethics are essential tools for improving care and management in health services and systems. Thus, this study aimed to analyze and evidence the ethical issues involving changes in the health work process, in the single health system, from the National Policy of Humanization (PNH) of 2003 and the National Curricular Guidelines (DCNs) for the undergraduate medical course of 2014. As a result, it was observed that bioethics was a fundamental tool for the establishment of new DCNs and $\mathrm{PNH}$, through the construction of new relations between users and workers and among them, with articulation of attitudes that guarantee an integral, decisive and humanized attention. In addition to helping physicians determine a link between scientific knowledge and humanistic knowledge, preventing the harms that technological advances can exert on living beings. It is concluded that bioethics was an important tool for the establishment of new DCNs and $\mathrm{PNH}$.

Keywords: Bioethics, medical education, health education, humanization of assistance. 


\section{Introdução}

As Diretrizes Curriculares Nacionais (DCNs), para o curso de graduação em Medicina de 2014, apresentaram competências para o perfil do formando egresso e, habilidades por eles desenvolvidas (Brasil, 2014). Da mesma forma, a Política Nacional de Humanização (PNH) de 2003 serviu de base para as alterações inovadoras nas graduações em saúde e no processo de trabalho em saúde das equipes multiprofissionais do Sistema Único de Saúde (SUS), com maior empatia e compaixão na atenção em saúde (Brasil, 2004).

A humanização do cuidado requer que este seja de forma integral e humana e, está prevista na $\mathrm{PNH}$. Estudos apontam que o cuidado humano corresponde a uma ação ética, em que as pessoas buscam conciliar suas aspirações e felicidade referentes ao seu bem-estar próprio em relação às obras praticadas em favor do conforto, segurança e saúde dos outros (Waldow, 2001). Para que isso ocorra, é necessário ao profissional de saúde, fazer uma reflexão crítica e contínua sobre o processo de trabalho, o qual define o modo como desenvolvem suas atividades profissionais ou a maneira como realizam o trabalho, suas habilidades e competências (Merhy, 2002).

A transformação da maneira como as atividades são desenvolvidas é uma característica marcante da humanidade e constitui uma parte central do sistema de desenvolvimento humano (Pasche, 2009). O grau de dificuldade da auto ponderação quantos aos atos praticados no ambiente laborativo, aumenta com a complexidade $\mathrm{e}$ com a indeterminação dos processos de trabalho. Quanto maior a complexidade do processo de trabalho e quanto menor a sistematização deste, maior dificuldade será encontrada ao se refletir sobre ele (Merhy, 2002).

O presente estudo intenciona fazer uma análise, sob o ponto de vista da bioética, e a partir de revisão sistemática da literatura, das influências da Política Nacional de Humanização e da promulgação das Diretrizes Curriculares Nacionais para o Curso de Graduação em Medicina de 2014, nas características do processo de trabalho em saúde do Sistema Único de Saúde (SUS), uma vez que, no período entre as DCNs de 2001 e as DCNs de 2014, houve a aprovação da Declaração Universal sobre Bioética e Direitos Humanos (DUBDH) divulgada pela Organização das Nações Unidas para a Educação, a Ciência e a Cultura (Unesco), em 2005, que deu ênfase à necessidade de o Estado incentivar a educação e a formação bioética em todos os níveis e esti- 
mular a difusão de informação e conhecimentos relacionados à bioética. Por isso, as DCNs de 2014 inserem no perfil do egresso médico formação generalista, humanista, crítica e reflexiva, capacitando-o a atuar pautado em princípios éticos (Carneiro; Porto, 2014).

\section{Metodologia}

\section{Estratégia de pesquisa e seleção}

As DCNs de 2014 e a PNH foram recuperadas, respectivamente, no portal do Ministério da Educação e no endereço eletrônico do Ministério da Saúde. Além disso, os artigos analisados foram selecionados nas bases de dados online Nescon, PubMed/Medline e Scielo no período de 2001 e 2019, utilizando os seguintes descritores e operadores booleanos "Educação Médica AND Bioética". E "Humanização da Assistência AND Bioética". A estratégia de busca baseou-se em três componentes: (i) Educação Médica, (ii) Bioética e (iii) Humanização da Assistência. Diante dos artigos selecionados, buscou-se o definir os artigos que estavam de acordo com os critérios de inclusão. Não houve restrição de linguagem na pesquisa de artigos. Estudos duplicados foram removidos comparando os autores, o título, o ano e o jornal de publicação. Todas as publicações relevantes foram baixadas e avaliadas.

\section{Características do estudo, critérios de exclusão e inclusão}

A exclusão dos artigos baseou-se em critérios bem definidos da seguinte forma: (i) Estudos abordando a educação médica após a $\mathrm{PNH}$, mas não demonstrando a contribuição da bioética, nesse processo (ii) Estudos que abordavam impactos da bioética na $\mathrm{PNH}$, mas não comentavam sobre as transformações na grade curricular do curso de Medicina ou sobre alguma transformação no processo de trabalho em saúde, (iii) Estudos de texto incompletos ou secundários (ou seja editoriais, comentários, cartas ao editor, dissertações, teses, capítulos de livros, publicações em anais de eventos e artigos com texto completo indisponível). Os critérios de inclusão foram: ser artigo original, e possuir análise sob o ponto de vista da bioética, das influências da PNH e das DCNs de 2014, em alguma característica do processo de trabalho em saúde do SUS.

\section{Extração dos dados}

Os dados foram extraídos dos artigos e organizados da seguinte maneira: I) Histórico das DCNs; II) DCNs 2001; III) PNH e a ética da responsabilidade; IV) As DCNs 
de 2014; V) O processo de trabalho em saúde e a bioética; VI) O código de ética médica; VII) A integração do ensino e serviço em saúde; VIII) Embasamento da bioética: a atenção à vida humana; IX) Contribuições das DCNs e PNH para a ética no processo de trabalho em saúde.

\section{Resultados e discussão}

\section{Histórico das DCNs}

Em 1981, o Programa de Integração Docente Assistencial (IDA) propôs importante iniciativa de inserção dos alunos na dinâmica de trabalho das unidades básicas de saúde. Surgiu como iniciativa do Ministério da Educação (ME) (Rezende, 2013). Para alguns autores, embora tenha se mostrado um avanço, a política ficou muito limitada aos serviços de saúde, com pouca repercussão nas mudanças curriculares (Pereira, 2013). Para que as transformações no ensino ocorressem, era necessário o surgimento de uma política pública de saúde (PPS). Por isso, é válido citar a Constituição da República Federativa do Brasil de 5 de outubro de 1988, a qual foi o marco inicial de uma nova ordem social, com a consolidação de um efetivo Estado Democrático Social de Direito, com a instituição de uma PPS, o SUS (Brasil, 1988).

No início dos anos 1990, o Projeto Uma Nova Iniciativa (PUNI), financiado pela Fundação Kellogg, foi pensado para superar algumas contradições dos Projetos IDA. Trouxe a lógica da multiprofissionalidade como orientação da formação e buscou superar a dicotomia entre os serviços de saúde e academia, presente nos projetos IDA, valorizando os serviços de saúde para a execução dos componentes curriculares. É importante notar que foram projetos relevantes, mas que no momento histórico (antes do SUS), contou com pouca atuação do Ministério da Saúde (MS) na regulação dos processos de formulação do modelo de formação profissional em saúde (Pereira, 2013).

Observando os avanços alcançados pela implantação dos projetos da década anterior, no início do século XXI, foi criada a Comissão Interministerial Nacional de Escolas Médicas (CINAEM), que teve uma atuação relevante para discussão da formação em medicina. Mas o cuidado inscrito nos modelos de atenção propostos pelo sistema era deixado como segunda opção até a publicação das Diretrizes Curriculares Nacionais para os cursos de graduação em saúde, incluindo a DCNs de 2001 (Maranhão, 2003). 
Além das DCNs, em 2002, o Programa Nacional de Incentivo a Mudanças Curriculares nos Cursos de Medicina (PROMED) foi implantado, promovendo importante discussão sobre a formação em Medicina de modo coerente com os princípios do SUS, estimulando a reforma curricular e a oferta de atividades de formação em hospitais universitários e serviços da atenção básica à saúde. O PROMED foi idealizado pelos Ministério da Saúde e da Educação e Organização Pan-Americana da Saúde (OPAS), com participação da Associação Brasileira de Educação Médica e a REDE UNIDA, facilitando a promulgação das DCNs de 2001, que consistiam em orientações a serem seguidas pelas instituições de ensino superior (IES) (Machado, 2005).

\section{DCNs 2001}

Em 18 de dezembro de 2001, na Universidade Federal de São Paulo (USP), o ME e o MS se reuniram para discutir as DCNs, que apresentam em seus primeiros artigos uma preocupação com a formação de recursos humanos para a área de saúde (Ceccim, Bilibio, 2002).

A proposta de criação das DCNs visava uma trajetória acadêmica mais elástica e os registros examinados para a construção das diretrizes foram a Constituição Federal de 1988, a LDB, o Relatório Final da $11^{\text {a }}$ Conferência Nacional de Saúde, Documentos da Organização Pan-americana de Saúde, da Organização Mundial de Saúde, da Rede Unida, a Declaração Mundial sobre Educação Superior no Século XXI da Unesco, publicações do ME e apetrechos que regem a prática dos profissionais de saúde. A finalização do documento para a regulamentação dos currículos dos cursos de graduação em saúde foi orientada pela Secretaria de Educação Superior do ME ao Conselho Nacional de Educação, que as legitimou (Pereira, 2013).

As DCNs de 2001 contemplavam a ética da responsabilidade e a cidadania, de forma semelhante à PNH (Cembranelli, 2010).

\section{PNH e a ética da responsabilidade}

Os debates a respeito da humanização desde a década de 1950, com propostas de reformulação dos modelos de atenção em saúde, receberam influências e contribuições da Declaração Universal dos Direitos Humanos; da defesa em prol da assistência ao parto humanizado - defesa na qual se identifica o movimento feminista como sujeito desse processo; da luta antimanicomial, e das demais críticas que vêm se juntar a essas, principalmente no que concerne ao modelo de assistência à saúde existente (Conceição, 2009). 
De um modo geral, as exigências da repercussões do processo de Reforma Sanitária apontavam a premência da estruturação de um sistema de saúde que não apenas assegurasse atendimento integral à totalidade dos cidadãos, mas entrada no SUS com características peculiares de qualidade, depreciando o modelo biomédico estritamente tecnicista/biologicista presente até então, caracterizado basicamente, pelos seguintes aspectos: i) Processo de trabalho em saúde desintegrado; ii) A centralização do poder; iii) A graduação médica distante da efetiva condição de saúde do povo, direcionada para o modelo hospitalocêntrico, relacionado ao aprimoramento tecnológico, circundado pela medicalização; iv) Esperas por atendimento em saúde demoradas; e v) Desinteresse por parte da gestão em dispensar recursos necessários para o setor de saúde.

Tais condições foram muito evidenciadas no fim da década de 1970 até a homologação da lei 8.080 de 1990 (Luz, 1991). Em 2003, a humanização passa a ser vista não como programa, mas como política pública, que além de tratar de dois pontos, a gestão e a atenção, atravessa/transversaliza as diferentes ações e instâncias gestoras do SUS, implicando em: i) Redução de filas e do tempo de espera; ii) Atendimento acolhedor e resolutivo baseado em critérios de risco; iii) Implantação de modelo de atenção com responsabilização e vínculo; iv) Garantia dos direitos dos usuários; v) Valorização do trabalho na saúde; vi) Gestão participativa nos serviços; vii) Política pública de caráter intersetorial; e viii) Fomento das grupalidades, coletivos e redes.

A política traz o debate de que a prática do cuidado se efetiva pelo encontro entre sujeitos, atuando uns com os outros, acolhendo intenções e expectativas e privilegiando a construção coletiva de uma experiência comum e solidária. Além disso, uma vez que ações consideradas desumanizadas na relação com os usuários do serviço público de saúde são percebidas, quanto ao aspecto cuidar, que deve ser resultante de ações como prevenir, proteger, tratar, recuperar e promover, é importante que o profissional de saúde consiga gerenciar o seu próprio processo de trabalho em saúde, objetivando melhorar a prestação de serviços e garantir um cuidado mais humanizado aos usuários do serviço de saúde em que atua (Oliveira, Collet, Vieira, 2006).

O destaque de projetos de humanização nos serviços de saúde, de acordo com a PNH, é percebido nas alterações no modelo de gestão e de atenção à saúde, orientadas pela ética da responsabilidade, objetivando a integralidade da assistência (Cembranelli, 2010). A ética da responsabilidade requer que as pessoas reflitam sobre o produto de seus atos, o que a torna facilitadora de transformações de hábitos 
de vida, de relacionamentos e de estabelecimento de laços sociais, ou seja, valoriza a inclusão social (Ayres, 2004).

Ética é uma palavra de origem grega "éthos", cujo significado é caráter, e a sua tradução para o latim é "mos", que corresponde a hábito. Em linhas gerais, a ética consiste no estudo da dicotomia bem e mal no agir humano e suas consequências (Moore, 1975; Padilha, 1995).

A análise da ética na área de estudos em ciências biológicas e similares denominam-se bioética, que significa "ética da vida" e que, atualmente, tem ocupado um espaço considerável nas discussões de questões de biodireito e de legislação, para que ocorram ações mais humanistas nos atendimentos médicos e nas pesquisas científicas com seres humanos (Oliveira, 2004). Além disso, a bioética valoriza a ponderação a respeito de demandas sociais e propositura para que se firmem alianças entre sociedade, cientistas, profissionais de saúde e governo sobre necessidades contemporâneas e projeções futuras (Ayres, 2004).

A bioética foi estruturada a partir de compreensões lógicas, propósitos e debates a respeito de ciência e vida e, no contexto do exercício profissional, esperam-se convicções éticas, fundamentadas na concepção de boa prática ou boa ciência, que sistematizem atitudes e condutas e previnam ações audaciosas e iatrogenias. Uma outra abordagem da bioética refere-se à formação das características peculiares do trabalhador de saúde, já que contribui para o aperfeiçoamento particularizado, o qual se somará à formação de caráter técnico-científico (Martins, 2008). Além disso, a bioética trata da relação dos humanos com outros seres vivos e meio-ambiente (Oliveira, 2004). Nesta perspectiva, considerando o conceito de bioética, pode ser citado o artigo 30 das DCNs de 2014, o qual define o perfil do egresso de Medicina voltado para a ética e ações humanísticas na prestação do cuidado aos clientes (Ferri; Gomes, 2015).

\section{As DCNs de 2014}

As DCNs de 2001 consistiam apenas em orientações para as instituições de ensino superior de Medicina, enquanto que as DCNs de 2014 são leis que determinam como deve ser a formação do estudante de Medicina. Isso tudo começou, devido ao fato de que após a aprovação da DUBDH divulgada pela Unesco em 2005, houve incentivo da intervenção estatal no ensino superior, com ênfase ao conteúdo de bioética e direitos humanos na formação universitária, a qual, em virtude disso, deve 
ser voltada à informação, reflexão e análise de questões e proposições relacionadas às diversas formas de desigualdades e suas implicações à saúde e à vida em suas diferentes abrangências, que incluem indivíduos, coletividades e o próprio planeta (Unesco, 2005).

Com isso, considerando a desigualdade de acesso aos serviços de saúde no Brasil, que tem como porta de entrada a atenção primária à saúde, foi necessária a promulgação da Lei do Mais Médicos (n0 12.871/2013) para recrutamento de mais recursos humanos médicos para o atendimento de populações residentes em locais desprovidos de profissionais de Medicina nos serviços do SUS (Carneiro; Porto, 2014). Além disso, a lei $12.871 / 2013$, prevê o fortalecimento da atenção básica e pesquisas direcionadas para o SUS (Brasil, 2014).

Essa necessidade de formação do profissional médico para atuação no SUS, fez com que as DCNs de 2014 valorizassem e preconizassem o ensino-serviço, considerando os três eixos básicos: i. Atenção em saúde; ii. Gestão em saúde; iii. Educação em saúde (Ferri; Gomes, 2015).

A literatura científica, bem como as DCNs de 2014, apresentaram a importância do contato de discentes de Medicina com a comunidade, para atender aos três eixos básicos preconizados, como sugerido por Carneiro e Porto (2014), que comentam que o corpo docente da faculdade de Medicina deve empregar, ao lecionar, metodologias ativas de ensino-aprendizagem, como o ensino de maneira multiprofissional e interdisciplinar.

A modalidade de ensino interdisciplinar encontra no ensino-serviço um alicerce para a construção de saberes, ou seja, a integração de diversas áreas das ciências da saúde. Isso, porque o ensino-serviço corresponde ao trabalho coletivo, como forma de aprendizagem, composto por acadêmicos, professores e trabalhadores que integram os serviços de saúde (que assumem a função de preceptoria) (Pereira Junior et al., 2015).

Acrescentamos ainda, o que diz Ivani Fazenda (1991), uma vez que afirma que a interdisciplinaridade está em constante ação, já que é a maneira de buscar conhecimento através de diferentes possibilidades.

O ensino-serviço permite ao estudante de medicina conviver nos diferentes serviços de saúde desde os primeiros períodos da faculdade. Ao analisarmos a presença do discente na Atenção Básica, percebemos, facilmente, que o mesmo realiza atividades de promoção da saúde prevista pela PPS (Vasconcelos, 2007). 
A graduação médica ao utilizar a modalidade de ensino-serviço permite a contextualização do aprendizado, que se faz necessária, no momento atual, para as ciências da saúde. Quanto mais contextualizada a disciplina com o processo de trabalho no SUS, maior a chance de que ocasione um aprendizado significativo, gerando motivação (Ferri; Gomes, 2015).

\section{O processo de trabalho em saúde e a bioética}

As condições de vida e de saúde das pessoas, suas famílias e a comunidade sofrem grande influência das ações do Estado e suas políticas. Além disso, o trabalho cotidiano de grande parte dos trabalhadores de saúde acontece no seio de uma grande política do Estado brasileiro: o SUS (Paim, Travassos, Almeida, Bahia et al.; 2011).

Além das leis específicas que regem os princípios de bioética e humanização, vale ressaltar, que o médico, em sua atividade, está sujeito à Constituição Federal de 1988, ao Código Civil de 2002, ao Código Penal, às leis trabalhistas e a outras Leis Estaduais e Municipais - preceitos gerais - e às regras que regulamentam a atividade médica, as quais são elaboradas pelo Ministério da Saúde, Conselho Nacional de Saúde, Conselho Federal de Medicina e Conselhos Regionais de Medicina, através de resoluções e portarias - diretrizes específicas. É válido ressaltar que há uma classificação, segundo graus de importância, entre as determinações e que nenhuma pode ser contrária à Constituição Federal (Souza, 2002).

Especificamente para que os princípios e diretrizes da PPS sejam atendidos, é importante que os trabalhadores dos serviços de atenção aos usuários sejam protagonistas neste processo (Starfield, 2002). De acordo com a Lei 8.080 de 19 de setembro de 1990 (Brasil, 1990), a lei que legitima o SUS, as ações e serviços públicos de saúde e os serviços privados contratados ou conveniados devem ser desenvolvidos de acordo com as diretrizes previstas na Constituição Federal de 1988, obedecendo a princípios organizativos e doutrinários, tais como: i) Universalidade de acesso aos serviços de saúde; ii) Integralidade de assistência, com prioridade para as atividades preventivas, sem prejuízo dos serviços assistenciais; iii) Equidade; iv) Descentralização político-administrativa com direção única em cada esfera de governo; v) Participação da comunidade; e vi) Regionalização e hierarquização.

Dessa forma, o processo de trabalho em saúde deve ser regido pelos direitos subjetivos previstos pela Lei Orgânica da Saúde (LOS), que se relacionam ao cuidado (Mattos, 2001). Isso pode ser proporcionado pela forma de organização dos 
serviços de saúde do SUS, já que favorece uma reorientação do processo de trabalho com maior potencial de aprofundar os princípios, diretrizes e fundamentos da PPS de ampliar a resolutividade e impacto na situação de saúde das pessoas e coletividades, além de propiciar uma importante relação custo-efetividade (Machado, 2005). Por isso, a ética deve ser aprendida pelo indivíduo, passando a fazer parte de sua personalidade, com consequente aplicação no cuidado em saúde (Toffler, 1984).

Para caracterizar o enfoque de assuntos relativos à vida, à morte e à saúde humana, que originam-se do convívio humano recorrente, bem como relacionamentos entre médicos e pacientes criou-se a expressão Bioética (Morin, 2001), cujo cerne é o modelo principialista (Potter, 1971).

\section{O código de ética médica}

Dentre as bases taxativas e fundamentais que se relacionam com a Bioética destaca-se o Código de Ética Médica (Toffler, 1984). Em 2010, o Código de Ética Médica, foi substituído, e os principais destaques foram: a letra legível ao redigir registro clínico em prontuário, receita e atestado médico, com identificação do registro profissional; o direito de escolha, uma vez que o médico deve apresentar todas as possibilidades terapêuticas - cientificamente reconhecidas - e aceitar a decisão do paciente; consentimento esclarecido emitido pelo paciente, diante de qualquer procedimento a ser realizado, salvo em caso de risco iminente de óbito; a recomendação de que o paciente não deve ser abandonado pelo seu médico; o profissional de saúde deve evitar procedimentos desnecessários, oferecendo todos os cuidados paliativos disponíveis, em caso de doenças incuráveis, levando sempre em conta a opção do paciente; o direito que o paciente tem de receber a cópia do seu prontuário e de obter uma segunda opinião e a ser referenciado a outro médico; Não é permita a participação do profissional médico em propagandas; em anúncios profissionais, é obrigatório incluir o número do Conselho Regional de Medicina; a receita não deve ser feita sem que o paciente passe por exame físico, seja por meio de veículo de comunicação ou internet; o profissional de saúde não pode ter relação com o comércio e a farmácia; mesmo após a morte do paciente, o sigilo médico deve ser mantido; é permitida a recusa laborativa em Medicina, nos locais inadequados à prestação de serviços e ações em saúde, com vistas ao cuidado integral, atendendo às normas de ambientação e biossegurança; o médico deve denunciar tortura; não é permitido ao médico vincular-se a cartões de descontos e consórcios, em especial na área de cirurgia plástica; abandonar plantão é falta grave; é vedada a participação do médico em manipulação 
genética e a escolha do sexo do bebê não pode ocorrer na reprodução assistida; o paciente deve decidir sobre os métodos contraceptivos que deseja usar (CFM, 2009).

Para inserir o graduando de Medicina na interação comunitária, com aplicação prática das normas do Código de Ética Médica, faz-se necessário a presença do preceptor em saúde, que em conjunto com a equipe multiprofissional, serve de base para a integração ensino-serviço (OMS, 1994).

\section{A integração do ensino e serviço em saúde}

A teia de integração ensino-serviço é estruturada entre os centros de ensino de graduação e as instituições de saúde, preferencialmente as públicas, e quem dá o suporte necessário é o preceptor em saúde, que consiste, geralmente, em um profissional do serviço de saúde, onde ocorrerão as atividades práticas (Starfield, 2002). A formação em Medicina deve ser orientada de acordo com os princípios do Sistema Único de Saúde. Para que isso ocorra de forma sustentável, a base da formação deve ser segundo o que consta, como comentado anteriormente, na Constituição Federal (1988), Art. 200. E na Lei nº 8.080 de 1990 (Pereira, 2013).

No processo de ensino e aprendizagem, os dois atores principais - docente e discente - adquirem conhecimento simultaneamente. Ambos devem construir as habilidades necessárias ao Sistema Único de Saúde, ao trocarem experiências, com aperfeiçoamento curricular e educação permanente em saúde, que a partir da $\mathrm{PNH}$ de 2003 e das DCNs de 2014, devem ser voltadas ao método clínico centrado na pessoa, o qual surgiu a partir das críticas do modelo focado na doença. São componentes do modelo que surge para uma maior atenção ao cuidado (Gusso, Lopes, 2012): i)Explorar a experiência da pessoa com a doença - os sentimentos em relação à doença; a percepção da pessoa sobre o que está acontecendo com ela, como a doença está a afetando; expectativas em relação ao médico, ao tratamento, ou à consulta; ii) Entender a pessoa como um todo - conhecer a história de vida da pessoa, a partir de uma anamnese bem feita; iii) Elaboração de um plano em conjunto de manejo dos problemas. É considerado o ponto central da do método centrado na pessoa, mostrando a aplicabilidade da valorização do sujeito; iv) Incorporação da prevenção e promoção da saúde; v) Intensificação da relação médico-paciente; e v) Ser realista.

Como resultados, a utilização do método tem proporcionado algumas melhorias nas ações de saúde, tais como: as consultas são agendadas de acordo com a triagem das necessidades dos pacientes, antecipando os atendimentos para aquelas pessoas, cujas condições clínicas e sociais requerem maior urgência; melhora do 
controle de doenças crônicas, como hipertensão e diabetes, bem como do quadro de saúde dos pacientes com sintomas inespecíficos; diminuição das queixas de má prática médica; redução dos sintomas em doenças mentais; menor prescrição de neurolépticos; menor solicitação de exames complementares; médicos mais satisfeitos; melhor relação médico-paciente; progresso na adesão ao tratamento; melhora dos desfechos neonatais, maior uso dos serviços preventivos; aperfeiçoamento dos cuidados paliativos (Carneiro; Porto, 2014).

O padrão de atendimento apresentado acima se aplica muito bem em todos os ambientes do Sistema Único de Saúde, sendo bastante válido para um cuidado integral e longitudinal, o que o torna essencial na atenção primária à saúde (Merhy, 2002). O aprendizado obtido no SUS é conseguido a partir da execução de atividades, que não se restringem a uma sucessão de ações feitas conforme as normas de biossegurança, mas corresponde à compreensão de que se é parte das políticas públicas, da sapiência e das habilidades desenvolvidas (Grosseman, Patrício, 2004).

\section{Embasamento da bioética: a atenção à vida humana}

Há diferentes recomendações para a composição dos preceitos que guiam os servidores de saúde na tomada de decisão, ao longo do percurso da carreira estudantil e laborativa. Porém, a principal orientação para a prática de ações em saúde é o fundamento ético da pessoa humana (Ayres, 2004).

As pessoas são seres singulares, dignas de respeitabilidade, formadas por diversos elementos, biológicos (abordados pelos estudos em saúde, Medicina, Enfermagem, Odontologia, Fisioterapia, por exemplo), condições mentais (os psicólogos abordam de forma mais esmiuçada), aspectos morais e sociais (melhor compreendidos pelas ciências sociais), espiritualidade (abordagem teológica). Portanto, devido a essas características evidenciadas, é possível afirmar que o ser humano é integral (Ceccim, Bilibio, 2002).

De modo semelhante ao que deve ocorrer com a pessoa, a vida humana deve ser respeitada, de acordo com a bioética. Para isso, deve-se estar atento à composição sucessiva, sequente, coordenada e gradativa da vida (Conceição, 2009). Faz-se necessário frisar que, diante da pessoa e vida humana, a ética abrange muitos fatores, que estão além da Deontologia - deveres e normas de conduta (Ayres, 2004). Por isso, é válido comentar que existem os princípios de análise bioética principialista, comumente utilizados e de grande aplicação na prática clínica na maioria dos países, introduzidos por Beauchamp e Childress, em 1989. Esses autores propõem quatro 
princípios bioéticos fundamentais: i) autonomia (respeito à singularidade), que trata do consentimento livre e esclarecido dos indivíduos-alvo e a proteção dos grupos vulneráveis e aos legalmente incapazes. Nesse sentido, a pesquisa envolvendo seres humanos deverá sempre tratá-los em sua dignidade, respeitá-los em sua autonomia e defendê-los em sua vulnerabilidade (Moore, 1975); ii) Beneficência (fazer o bem): ponderação entre os riscos e benefícios, tanto atuais como potenciais, individuais e coletivos, comprometendo-se com o máximo de benefícios e o mínimo de danos e riscos; iii) Não maleficência (não fazer o mal): garantia de que danos previsíveis serão evitados (eutanásia passiva em doente terminal, por exemplo) (Vasques, 2000); iv) Justiça e Equidade: realce social da pesquisa com vantagens significativas para os sujeitos participantes e minimização do ônus para os mais vulneráveis, garantindo a igual consideração dos interesses envolvidos, sem perder o sentido de sua destinação sócio-humanitária (Ligiera, 2005).

Como todos os profissionais da área de saúde estabelecem relações com outras pessoas, sobretudo pacientes, as DCNs de 2014 reforçando a necessidade de orientações do graduando quanto ao processo de trabalho no SUS, realça a proximidade da graduação em Medicina com os princípios da PPS, o desenvolvimento da formação humanista e o atendimento às questões propostas pela DUBDH, com a atenção integral no centro da grade curricular, para que as ações de saúde sejam de melhor qualidade (Ferri; Gomes, 2015).

\section{Contribuições das DCNs e PNH para a ética no processo de trabalho em saúde}

O trabalhador em saúde e o acadêmico de Medicina, após as recomendações das DCNs de 2014, precisam abordar o paciente com empatia, considerando o indivíduo de forma integral. A fim de atender a esse objetivo, deve-se valorizar o trabalho interdisciplinar e multiprofissional. Além disso, a relação médico-paciente necessita manter os dados obtidos na consulta sob sigilo (Ferri; Gomes, 2015).

Uma vez que se tenha em mente a necessidade de se colocar no lugar do paciente antes de qualquer coisa, salienta-se que as mudanças que ocorreram em saúde, com mercantilização da mesma, correspondem a um grande desafio para a gestão. Sempre que uma nova terapêutica é anunciada, a população quer adquiri-la (Ceccim, Bilibio, 2002). Devido a isso, muitos pacientes que não necessitam de planos terapêuticos mais complexos, acabam requisitando que isso seja prescrito pelo médico. Porém, para o rastreamento e intervenções precoces, deve-se considerar os riscos e 
benefícios da terapêutica, para que não haja iatrogenia. O nome que é dado a essa ponderação, é prevenção quaternária (Gusso, Lopes, 2012).

Um bom momento para oportunizar a integração de medidas preventivas e curativas é o acolhimento. Com a Política Nacional de Humanização, que valoriza o acoIhimento, iniciou-se uma transformação do modelo biomédico para o cuidado integral (Brasil, 2004). Para isso, é preciso que todos os aspectos da vida do paciente sejam considerados (biológico, social, psicológico, teológico), o que requer aliar o conhecimento técnico-científico à humanização (Conceição, 2009).

Após o estabelecimento de plano diagnóstico ou terapêutico, objetivando atender à integralidade em saúde, o esclarecimento com subsequente consentimento, por parte do usuário do sistema de saúde, consiste em etapa importante da consulta e também a um desafio ético. O profissional de saúde deve se manter atento ao fato de que, por ser uma pessoa complexa e única, o paciente pode estar ansioso, receoso, aflito, impaciente, sensação desagradável e/ou em sofrimento, o que pode restringir sua autonomia. Portanto, nessa fase do atendimento, o médico deve estar preparado para a educação em saúde, que consiste em orientar o usuário do serviço de saúde quanto ao seu problema de saúde, métodos diagnósticos e tratamento, de forma compreensível, mantendo-se disponível para eventuais dúvidas que possam surgir (Ayres, 2004).

As habilidades de comunicação e formação da relação médico-paciente devem tomar como base a necessidade de escuta que o profissional de saúde deve apresentar. Não basta apenas informar ao paciente sobre sua condição de saúde, prognóstico, expectativas da terapêutica, mas ouvir atentamente tudo o que ele tem a dizer, uma vez que as suas principais necessidades serão percebidas durante o relato e as queixas referidas pelo paciente. É também quando se estabelece um diálogo efetivo, com comunicação bilateral, que indivíduo consegue elucidar suas dúvidas e demonstrar alguma fragilidade social, ou impedimentos de adesão à terapêutica (Grosseman, Patrício, 2004).

De posse das informações obtidas durante a consulta, o sigilo e a confidencialidade dos dados deve ser mantida. O registro da anamnese deve ser feito em prontuário do paciente, o qual não deve ser manuseado por pessoas que não manterão os assuntos em segredo. Além do mais, comentários não devem ser feitos referentes às situações relatadas ou observadas, seja dentro do consultório ou local de atendimento, ou ainda, em domicílio, quando o paciente for avaliado em sua residência ou em locais externos ao serviço de saúde (Gusso, Lopes, 2012). 
Os prontuários são documentos, que quando preenchidos adequadamente, com anotações adequadas referentes ao exame clínico - anamnese somada ao exame físico - obtido durante a consulta, permitem o seguimento e avaliação do usuário do serviço de saúde, tal como possibilita a obtenção de informações referentes à qualidade do atendimento prestado. Além disso, o registro clínico possibilita o acompanhamento longitudinal do paciente. Mas, o referido documento médico, apesar de ficar sob os cuidados do estabelecimento de saúde ou do próprio profissional, pertence ao cliente. Por isso, as informações colhidas e apontadas na ficha de atendimento, não podem ser disponibilizadas para outras pessoas, nem mesmo familiares, sem o consentimento do paciente, a não ser que seja solicitado sob mandato judicial. Essa norma é válida tanto para o prontuário de papel, escrito manualmente, como para o registro eletrônico, que diante do avanço tecnológico e dos novos hábitos adquiridos pela sociedade tem sido muito utilizado pelos serviços de saúde (Pasche, 2009).

Diante das diferentes informações divulgadas pelos meios de comunicação, que tiveram expressiva melhora com os aperfeiçoamentos da tecnologia, as pessoas por conviverem em sociedade devem ter oportunidades para obter autonomia intelectual para decidir sobre o que querem para si, o que deve ser respeitado por todos, mesmo aquelas condições que podem levar a algum prejuízo de saúde, como o hábito etílico ou tabagista. Porém, essas decisões particulares devem ser sensatas, o que requer maturidade pessoal que demanda conhecimento e formação para decisão com um critério pertinente. Por isso, quando a liberdade individual proporciona um estilo de vida insalubre e que pode trazer danos a outras pessoas, cabe ao Estado intervir em prol da coletividade (Vasques, 2000).

Nem sempre os hábitos de vida não saudáveis (sedentarismo, etilismo, tabagismo, por exemplo) trarão problemas de saúde para outras pessoas, mas somente para o cliente. Nessas condições, não deve ser feito juízo de valor, com segregação desses usuários do serviço de saúde pública. Por isso, deve ser incentivada a educação popular em saúde, mas não a imposição. E a bioética aponta para a reflexão sobre os problemas enfrentados diante das rupturas de paradigmas da saúde, de uma garantia para uma obrigação, tal qual as dificuldades para aceitação dessa mudanças (Pereira, 2013).

Estudos apontam ainda, que a ponderação pelos profissionais de saúde diante das ações prestadas e da relação médico-paciente, com aperfeiçoamento contínuo em favor da obtenção de uma maior satisfação do usuário, reduz o número de processos individuais, melhora da adesão à terapêutica, com consequências positivas na saúde da população (Lampert, 2002). 


\section{Considerações finais}

As Diretrizes Curriculares Nacionais para o curso de Medicina de 2014 reforçam a necessidade de orientar a formação de recursos humanos a partir de atividades práticas no Sistema Único de Saúde, valorizando a aproximação da graduação médica aos princípios da PPS, com a realização do ensino-serviço pautado pelos princípios da bioética, com ampliação da formação humanista, conforme proposto, em 2003, pela Política Nacional de Humanização, dando ênfase à integralidade como eixo norteador das ações prestadas em saúde.

As novas DCNs e PNH reforçam a necessidade de construção, de forma coletiva e compartilhada, das mudanças necessárias, por meio das quais os trabalhadores possam reinventar o seu processo de trabalho. Sendo necessário ao profissional de saúde reconhecer e ser sensível às peculiaridades e vulnerabilidades de cada cidadão, de forma colaborativa para que ocorra redução das filas de espera por atendimentos, no Sistema Único de Saúde. É essencial que o usuário possa tomar parte, com autonomia e participando da gestão da unidade, tendo liberdade para inclusão da sua rede sócio-familiar no processo do cuidar, e, assim, ser corresponsabilizado pelo cuidado que lhe é prestado.

O modelo de graduação em Medicina proposto em 2014 combate as metodologias tradicionais de ensino-aprendizagem com vistas ao desenvolvimento de habilidades e competências de profissionais-cidadãos envolvidos com a restauração indispensável no processo de trabalho em saúde: o cuidado para a resolução das necessidades em saúde da sociedade.

\section{Referências}

1. Ayres JRCM. Cuidado e reconstrução das práticas de saúde. Revista Interface, comunicação, saúde e educação 2004; 14(8): 73-92.

2. BRASIL. Dispõe sobre as condições para a promoção, proteção e recuperação da Saúde, a organização e o funcionamento dos serviços correspondentes e dá outras providências. Brasília: Lei n. 8.080, de 19 de setembro de 1990; 1990.

3. BRASIL. HumanizaSUS: Política Nacional de Humanização: Documento base para gestores e trabalhadores do SUS. Brasília: Ministério da Saúde/ Secretaria Executiva /Núcleo Técnico da Política Nacional de Humanização; 2004.

4. BRASIL. Constituição da República Federativa do Brasil de 1988. Brasília: Presidência da República; 1988. 
5. BRASIL. CONSELHO NACIONAL DE EDUCAÇÃO/CÂMARA DE EDUCAÇÃO SUPERIOR. Parecer n. 116 de 03 de abril de 2014. Diretrizes Curriculares Nacionais do Curso de Graduação em Medicina. Brasília, DF. Acessível em: http://portal.mec.gov.br/index.php?option=com_docman\&view=download\&alias=15874-rces003-14\&category_slug=junho-2014-pdf\&ltemid=30192

6. Carneiro LA, Porto CC. Saúde mental nos cursos de graduação: interfaces com as diretrizes curriculares nacionais e com a reforma psiquiátrica. Cadernos Brasileiros de saúde Mental 2014; 6(14): 150-167.

7. Ceccim R, Bilibio LFS. Articulação com o movimento estudantil da área da saúde: uma estratégia de inovação na formação de recursos humanos para o SUS. In: Ferla AA, Fagundes SMS. Tempo de inovações: a experiência da gestão da saúde do Rio Grande do Sul. Porto Alegre: Dacasa; 2002: 163-174.

8. Cembranelli, F. Um projeto de humanização: para que, para quem? 2010. Acessível em: www.portalhumaniza.org. $\mathrm{br} / \mathrm{ph} / \mathrm{texto}$. asp?id=49.

9. Conceição TS. A Política Nacional de Humanização e suas implicações para a mudança do modelo de atenção e gestão na saúde: notas preliminares. SER Social 2009; 11(25): 194-220. Acessível em: http://seer.bce.unb.br/index.php/ SER_Social/article/viewFile/373/226.

10. CONSELHO FEDERAL DE MEDICINA. Código de Ética Médica 2009: Resolução CFM no 1.931/2009. Brasília: CFM; 2009.

11. Ferri $P$, Gomes $R$ Formação situada ou situações do formar: internato médico em questão. Revista Brasileira de Educação Médica 2015; 39(2): 252-260.

12. Grosseman S, Patrício ZM. A relação médico-paciente e o cuidado humano: subsídios para a formação da educação médica. Revista Brasileira de Educação Médica 2004; 28 (2): 99-105.

13. Gusso G, Lopes JMC. Tratado de medicina de família e comunidade: princípios, formação e prática. Porto Alegre: Artmed; 2012.

14. Lampert J. Tendências de mudanças na formação médica no Brasil: tipologia das escolas. São Paulo: Hucitec; 2002.

15. Ligiera, WR. Os princípios da bioética e os limites da atuação médica. Revista Ibero Americana de Direito Público. Rio de Janeiro (Coord. Martins, IGS), ano 5 , n. 20, p.410-27, $4^{\circ}$ trim.; 2005.

16. Luz MT. Notas sobre as políticas de saúde no Brasil de "transição democrática" - Anos 80. PHYSIS -Revista de Saúde Coletiva 1991; 201-216. Acessível em: http://www.scielo.br/pdf/physis/v1n1/04.pdf.

17. Machado CV. Direito universal, política nacional: o papel do Ministério da Saúde na política de saúde brasileira de 1990 a 2002 (Tese). Rio de Janeiro: Instituto de Medicina Social da Universidade do Estado do Rio de Janeiro; 2005. 
18. Maranhão EA. Construção coletiva das Diretrizes Curriculares Nacionais dos Cursos de Graduação da Saúde: uma contribuição para o Sistema Único de Saúde. In: Almeida MJ. Diretrizes Curriculares Nacionais para os cursos universitários da área de saúde. Londrina: Rede Unida; 2003.

19. Martins MA. Novas tendências do ensino médico. Gaz. Méd. Bahia. 2008; 78(1): 22-24.

20. Mattos RA. Os sentidos da integralidade: algumas refexões acerca de valores que merecem ser defendidos. In: Pinheiro, Roseni e Mattos, Ruben. Os 64 Curso de Especialização - Saúde da Família Módulo 3 - Políticas de Saúde sentidos da integralidade na atenção e no cuidado à saúde. Rio de Janeiro: UERJ - IMS ABRASCO; 2001.

21. Merhy EE. Saúde: a cartografia do trabalho vivo. São Paulo: Hucitec; 2002.

22. Moore GE. Princípios éticos. São Paulo: Abril Cultural; 1975.

23. Morin E. A cabeça bem feita. Rio de Janeiro: Bertrand-Brasil; 2001.

24. Oliveira BRG, Collet N, Vieira CS. A humanização na assistência à saúde. Rev Latino-am Enfermagem 2006; 14 (2): 277-284. Acessível em: https://www.scielo. $\mathrm{br} / \mathrm{pdf} / \mathrm{rlae} / \mathrm{v} 14 \mathrm{n} 2 / \mathrm{v} 14 \mathrm{n} 2 \mathrm{a} 19 . \mathrm{pdf}$.

25. Oliveira F. Bioética, uma face da cidadania. $8^{\mathrm{a}}$ impressão. $2^{\mathrm{a}}$ ed. Reformulada, São Paulo: Moderna; 2004.

26. OMS. Como tornar a prática e a educação médicas mais adequadas às necessidades de saúde da população: a contribuição do médico de família. WONCA 1994. Acessivel em: https://www.globalfamilydocotor/publications/.

27. Padilha MICS. Questões éticas: cuidados metodológicos na pesquisa de enfermagem. Texto Contexto Enferm. 1995; 4(2): 118-32.

28. Paim J, Travassos C, Almeida C, Bahia L, Macinko J. O sistema de saúde brasileiro: história, avanços e desafios. The Lancet 2011; 11-31. Acessivel em: https:// actbr.org.br/uploads/arquivo/925_brazil1.pdf.

29. Pasche DF. Política nacional de humanização como aposta na produção coletiva de mudança nos modos de gerir e cuidar. Interface: Comunicação, Saúde, Educação 2009; 13(1): 701-708.

30. Pereira IDF. A formação profissional em saúde no Brasil pós 1988: aspectos das Conferências Nacionais de Saúde e das Políticas Públicas (Dissertação). Rio de Janeiro: Escola Nacional de Saúde Pública; 2013.

31. Pereira Junior GA et al. $O$ ensino de urgência e emergência de acordo com as novas diretrizes nacionais e a Lei dos Mais Médicos. Caderno ABEM 2015; 11: 20-47.

32. Potter VR. Bioethics: bridge to the future. Englewood Cliffs: Prentice Hall; 1971.

33. Rezende M. A articulação educação saúde (AES) no processo de formulação das políticas nacionais voltadas a formação de nível superior dos profissionais de saúde (Tese). Rio de Janeiro: Escola Nacional de Saúde Pública; 2013. 
34. Santos A. Des-construindo a didática. Revista Universidade Rural, série Ciências Humanas. Rio de Janeiro. Vol. 23, N 01; 2001. Acessível em: http://www.ufrrjleptrans.hpg.ig.com.Br/mdes.htm.

35. Souza RR. O Sistema Público de Saúde. Brasília; ministério da Saúde; 2002.

36. Starfield B. Atenção primária: equilíbrio entre necessidades de saúde, serviços e tecnologia. Brasília: UNESCO/ Ministério da Saúde; 2002.

37. Toffler A. Science and change. In: Prigogine I, Stengers I. Order out of chaos. Toronto: Bantam; 1984.

38. Unesco. Declaração Universal sobre Bioética e Direitos Humanos. Paris: UNESCO; 2005.

39. Vasconcelos EM. Educação popular: instrumento de gestão participativa dos serviços de saúde. In: Ministério da Saúde. Secretaria de Gestão Estratégica e Participativa. Departamento de apoio à Gestão Participativa. Caderno de educação popular e saúde. Brasília (DF): Ministério da Saúde; 2007.

40. Vasques AS. Ética. Rio de Janeiro: Civilização Brasileira; 2000.

41. Waldow VR. Cuidado humano: o resgate necessário. Porto Alegre: Sagra Luzzatto; 2001.

Recebido: 25/06/2019. Aprovado: 04/09/2019. 